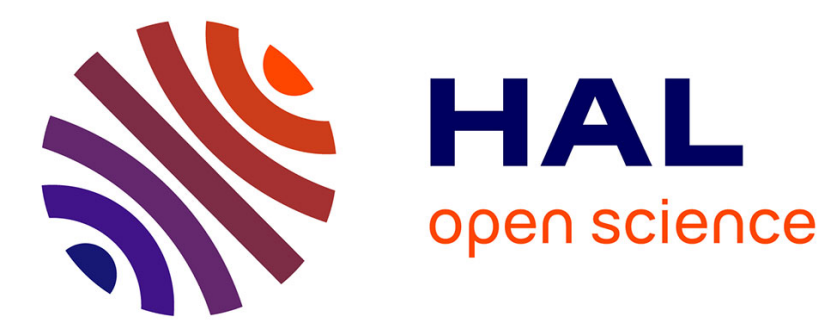

\title{
The plasmasheath transition in low temperature plasmas: on the existence of a collisionally modified Bohm criterion
}

R P Brinkmann

\section{- To cite this version:}

$\mathrm{R} \mathrm{P}$ Brinkmann. The plasmasheath transition in low temperature plasmas: on the existence of a collisionally modified Bohm criterion. Journal of Physics D: Applied Physics, 2011, 44 (4), pp.42002. 10.1088/0022-3727/44/4/042002 . hal-00589768

\section{HAL Id: hal-00589768 https://hal.science/hal-00589768}

Submitted on 2 May 2011

HAL is a multi-disciplinary open access archive for the deposit and dissemination of scientific research documents, whether they are published or not. The documents may come from teaching and research institutions in France or abroad, or from public or private research centers.
L'archive ouverte pluridisciplinaire HAL, est destinée au dépôt et à la diffusion de documents scientifiques de niveau recherche, publiés ou non, émanant des établissements d'enseignement et de recherche français ou étrangers, des laboratoires publics ou privés. 


\title{
The plasma-sheath transition in low temperature plasmas: On the existence of a collisionally modified Bohm criterion
}

\author{
R.P. Brinkmann \\ Institute for Theoretical Electrical Engineering, \\ Ruhr University Bochum, D-44780 Bochum, Germany
}

(Dated: November 30, 2010)

\begin{abstract}
The plasma-sheath transition in stationary low temperature plasmas is investigated for arbitrary levels of collisionality. The model under study contains the equations of continuity and motion for a single ion species, Boltzmann's equilibrium for the electrons, and Poisson's equation for the field. Assuming that the electron Debye length $\lambda_{\mathrm{D}}$ is small compared to the ion gradient length $l=n_{\mathrm{i}} / \frac{\partial n_{\mathrm{i}}}{\partial x}$, a first order differential equation is established for the ion density $n_{\mathrm{i}}$ as function of the transformed spatial coordinate $q=\int n_{\mathrm{i}} d x$. A characteristic feature of this novel 'sheath equation' is an internal singularity of the saddle point type which separates the depletion-field dominated sheath part of the solution from the ambipolar diffusion controlled plasma. The properties of this singularity allow to define, in nonarbitrary way, a collisionally modified Bohm criterion which recovers Bohm's original expression in the collisionless limit but remains meaningful also when collisions are included. A comparison is made with the collisionally modified Bohm criteria proposed by Godyak [V.A. Godyak, Phys. Lett. 89A, 80 (1982)], Valentini [H.-B. Valentini, Phys. Plasmas 3, 1459 (1996)] and Chen [X.P. Chen, Phys. Plasmas 5, 804 (1997)] as well as with the approaches of Riemann [K.-U. Riemann, J. Phys. D; Appl. Phys. 24, 493 (1991)] and Franklin [R.N. Franklin, J. Phys. D: Appl. Phys. 36, 2821 (2003)] who argued that the definition of a collisionally defined Bohm criterion is not possible.
\end{abstract}

PACS numbers: 52.40.Kh 
The subdivision of a gas discharge into the plasma bulk and the sheath was introduced by Langmuir in 1928 [1]. Together with Tonks he also coined the term sheath edge for the separation of the two "rather distinct" zones [2]. (In the standard 1D geometry, bulk and sheath are intervals while the sheath edge is a point.) The connection of the two zones was first studied by Bohm who, in 1949, formulated his famous "criterion for a stable sheath" [3]: To ensure nonoscillatory solutions, ions must enter the sheath from infinity with a minimum speed (the 'Bohm speed') $v_{\mathrm{B}}=\sqrt{T_{\mathrm{e}} / m_{\mathrm{i}}}$. (Here, $m_{\mathrm{i}}$ denotes the ion mass and $T_{\mathrm{e}}$ the temperature of the electrons in energy units, i.e., $k_{\mathrm{B}} \equiv 1$.)

The Bohm criterion $\left|v_{\mathrm{i}}\right| \geq v_{\mathrm{B}}$ has vexed the plasma community for more than four decades. One of its mysteries was that it could not only be derived from Bohm's original sheath equations (collisionless ions plus Poisson's equation), but also from a "presheath model" (collisional ions plus quasineutrality); there, however, from the properties of a singularity [4]. A systematic reconstruction of the Bohm criterion was given in 1991 by Riemann who investigated the plasma-sheath transition under the condition that the Debye length $\lambda_{\mathrm{D}}$ is small compared to the mean free path $\lambda[5]$. Asymptotically matching the sheath (scale $\lambda_{\mathrm{D}}$ ) and the presheath (scale $\lambda$ ) by a transition layer (scale $\lambda_{D}^{4 / 5} \lambda^{1 / 5}$ ), he found a solution uniformly valid on all scales and showed that the Bohm criterion is marginally fulfilled.

Riemann's analysis of the regime $\lambda_{\mathrm{D}} / \lambda \ll 1$ motivated research into what happens when the scale ratio is large. It proved difficult: Some researchers who studied the plasma-sheath transition in the presence of collisions found that the Bohm criterion must be modified [6-10]. Others objected: Riemann stressed that "there is no reason and no basis to formulate a new modified Bohm criterion accounting for collisions in the sheath" and that the "various heuristic attempts" are "inconsistent and lead to unreasonable results" [11]. Franklin went along: "There is no such thing as a collisionally modified Bohm criterion" [12].

This manuscript aims to take part in that discussion by offering an alternative perspective. A standard sheath model will be analyzed, similar to the ones investigated in refs. [6-12]. As in [11] and [12], the Debye length $\lambda_{\mathrm{D}}$ will be treated as small compared to a certain other scale of the dynamics. However, that 'other scale' will not be the ion mean free path $\lambda$ but the ion density gradient length $l=n_{\mathrm{i}} /\left|\frac{\partial n_{\mathrm{i}}}{\partial x}\right|$. Employing arguments that are not heuristic, it will be shown that the assumption $\lambda_{\mathrm{D}} \ll l$ implies - for all ratios $\lambda_{\mathrm{D}} / \lambda$ - the existence of a certain mathematical structure (a removable singularity) with properties that allow to unambiguously define a 'collisionally modified Bohm criterion'. 
As stated, the model under study is quite conventional. A one-dimensional Cartesian geometry is assumed, described by an $x$-axis pointing from the electrode $x_{\mathrm{E}}=0$ to the bulk. The field is related to the ion and electron densities via Poisson's equation, with the electrons obeying Boltzmann's relation with an electron temperature $T_{\mathrm{e}}$ :

$$
-\epsilon_{0} \frac{\partial^{2} \Phi}{\partial x^{2}}=\epsilon_{0} \frac{\partial E}{\partial x}=e\left(n_{\mathrm{i}}-n_{0} \exp \left(\frac{e \Phi}{T_{\mathrm{e}}}\right)\right) .
$$

Ionization is neglected so that the ion flux density $\Psi_{\mathrm{i}}$ to the electrode is spatially constant. The equation of motion takes into account acceleration by the electrical field and friction due to charge exchange and elastic collisions with the neutrals:

$$
\begin{aligned}
& n_{\mathrm{i}} v_{\mathrm{i}}=-\Psi_{\mathrm{i}}, \\
& m_{\mathrm{i}} v_{\mathrm{i}} \frac{\partial v_{\mathrm{i}}}{\partial x}=e E-\frac{\left|v_{\mathrm{i}}\right|}{\lambda} m_{\mathrm{i}} v_{\mathrm{i}} .
\end{aligned}
$$

The mean free path $\lambda$ depends on the velocity of the ions. In many gases, it scales like $\left|v_{\mathrm{i}}\right|$ for small $v_{\mathrm{i}}$ (Langevin interaction) and is constant for large $v_{\mathrm{i}}$ (charge exchange interaction and 'hard sphere' elastic collisions). In argon, literature results are well reproduced by $\lambda\left(v_{\mathrm{i}}\right)=\left|v_{\mathrm{i}}\right| / n_{\mathrm{N}} \sigma_{0} \sqrt{v_{0}^{2}+v_{\mathrm{i}}^{2}}$ where $n_{\mathrm{N}}$ is the gas density and $\sigma_{0}=10^{-18} \mathrm{~m}^{2}$ and $v_{0}=550 \mathrm{~m} / \mathrm{s}$. The system is completed by the conditions of neutrality and transport equilibrium for $x \rightarrow \infty$ and the specification of a potential or current at the electrode location $x_{\mathrm{E}}$.

For comparison, it is advantageous to first study the model without any simplifications. Fig. 1 shows solutions which cover the range from a collisionless $(p=0.1 \mathrm{~Pa})$ to a collision dominated sheath $(p=100 \mathrm{~Pa})$. The ion flux is $\Psi_{\mathrm{i}}=2.6 \times 10^{18} / \mathrm{m}^{2} \mathrm{~s}$, the electron temperature is $T_{\mathrm{e}}=3 \mathrm{eV}$. All curves exhibit a smooth transition from electron depletion to quasineutrality (= from sheath to plasma) without any pecularities at the Bohm speed $v_{\mathrm{B}}$ or the density $n_{\mathrm{B}}=\Psi_{\mathrm{i}} / v_{\mathrm{B}}$. This, of course, is not surprising; the Bohm criterion is absent in the unabridged model and appears only within asymptotic regimes [11, 12].

The standard asymptotic regime in the investigation of the Bohm criterion is ' $\lambda_{\mathrm{D}} \ll \lambda$ '. Bohm himself studied the situation on the scale $x \sim \lambda_{\mathrm{D}}$. Alternatively, one can focus on the scale $x \sim \lambda$ and employ the 'plasma approximation': Substituting quasineutrality $\left(n_{\mathrm{e}}=n_{\mathrm{i}}\right)$ for Poisson's equation and combining it with Boltzmann's relation yields $E=-\frac{T_{\mathrm{e}}}{e} \frac{1}{n_{\mathrm{i}}} \frac{\partial n_{\mathrm{i}}}{\partial x}$. Inserting this 'ambipolar field' into the equation of motion and utilizing flux conservation leads to the 'preseath model' (here formulated in $n_{\mathrm{i}}$ instead of the ususal $v_{\mathrm{i}}$ ):

$$
\left(\frac{T_{\mathrm{e}}}{m_{\mathrm{i}}}-\frac{\Psi_{\mathrm{i}}^{2}}{n_{\mathrm{i}}^{2}}\right) \frac{1}{n_{\mathrm{i}}} \frac{\partial n_{\mathrm{i}}}{\partial x}=\frac{1}{\lambda} \frac{\Psi_{\mathrm{i}}^{2}}{n_{\mathrm{i}}^{2}}
$$


Solutions of the presheath model are also shown in fig. 1, calculated for the same data set as the exact solutions to which they asymptotically converge. All have a singularity at the density $n_{\mathrm{B}}=\Psi_{\mathrm{i}} / v_{\mathrm{B}}$ and the speed $v_{\mathrm{B}}$, regardless of the pressure and the collision model. However, it is only for small values of $\lambda_{\mathrm{D}} / \lambda$ that this singularity (the 'Bohm point') has any relation to the unabridged model: With decreasing $\lambda$, the Bohm point moves from the bulk side of the sheath (where quasineutrality applies) to the center (where quasineutrality fails). The ambipolar field ceases to be a valid approximation and the presheath solutions begin to deviate from their exact counterparts. Riemann's interpretation of this situation was that with increasing collisionality the "Bohm criterion loses gradually its significance" [11].

However, could that be an over-interpretation? Strictly stated, what loses significance is only the ' $\lambda_{\mathrm{D}} \ll \lambda$ approach' to the Bohm criterion, for instance Riemann's own asymptotic analysis in $\lambda_{\mathrm{D}} / \lambda$ and the uniform approximation based on it. That does not prove that there is no other possibility to reconstruct the Bohm criterion, i.e., to unambiguously single out a certain point in the gradual plasma-sheath transition as the 'sheath edge'. If such a point could be constructed without resorting to the assumption $\lambda_{\mathrm{D}} \ll \lambda$, the resulting 'Bohm criterion' would not lose its significance at higher collisionality.

How could that be done? Any conceivable 'Bohm criterion' should mark the transition from the ambipolar diffusion dynamics of the bulk to the unipolar motion in the sheath. In the $\lambda_{\mathrm{D}} \ll \lambda$ 'presheath approach' it describes the singularity which occurs when inertia equals the ambipolar field and the prefactor of $\frac{\partial v_{\mathrm{i}}}{\partial x}$ vanishes. A finite $\lambda_{\mathrm{D}} / \lambda$ model, in contrast, should not terminate but merge into a solution for the sheath. Necessarily then $n_{\mathrm{e}} \neq n_{\mathrm{i}}$ and the depletion field must enter the picture. In other words, a more general expression for the field is needed which, however, must still be formulated in terms of the local ion density and its spatial derivative: Otherwise, it could not be combined with the ion inertia term.

Seemingly, such a generalized but local representation of the electric field is impossible: Physically, the field is local only in the quasineutral zone where the ambipolar formula holds; in the depletion region it is not local but dependent on the spatial integral of the ion density. However, this problem can be overcome: What is really required is not physical locality but only mathematical locality, i.e., locality in a transformed coordinate $q(x)$. The key idea is to take as the new coordinate the spatial integral of the ion density itself, $q(x)=\int n_{\mathrm{i}}(x) d x$. The field in the depletion region can be then expressed as a linear function on $q$, while the field in the ambipolar zone remains proportional to a derivative of $n_{\mathrm{i}}$. 
In two recent publications $[13,14]$ the present author has spelled out the details of such a field construction: First, the equivalent electron edge $s$ is defined to be the point where the integrated electron charge below is just equal to the net charge density above:

$$
\int_{x_{\mathrm{E}}}^{s} n_{\mathrm{e}}\left(x^{\prime}\right) d x^{\prime}=\int_{s}^{\infty} n_{\mathrm{i}}\left(x^{\prime}\right)-n_{\mathrm{e}}\left(x^{\prime}\right) d x^{\prime}
$$

The Boltzmann-Poisson equation is then approximately solved under the assumption that the transition from electron depletion to quasineutrality - which takes place within a few Debye lengths around $s$ - is fast compared to the gradient length of the ion density $l=n_{\mathrm{i}} / \frac{\partial n_{\mathrm{i}}}{\partial x}$. Technically, separate expansions are established, respectively, for the unipolar zone $x \ll s$, the transition zone $|x-s| \lesssim \lambda_{\mathrm{D}}$, and the ambipolar zone $x \gg s$. The expansions are systematically carried out up to the order where the ambipolar field appears in the zone $x \gg s$, and then spliced together by the 'asymptotic matching principle'. The last step of the construction is an approximate transformation, again employing $\lambda_{\mathrm{D}} \ll l$, of the field expression into the 'charge coordinates' defined as

$$
q(x)=\int_{s}^{x} n_{\mathrm{i}}\left(x^{\prime}\right) d x^{\prime}
$$

The resulting formula for the field, termed the advanced algebraic approximation $(A A A)$, can be seen as an improvement of Godyak's well-known step model [15]. (It reduces to that model in the limit $T_{\mathrm{e}} \rightarrow 0$.) The AAA is a mathematically local representation of the field in terms of the charge coordinate $q$, the ion density $n_{\mathrm{i}}$, and the derivative $\frac{\partial n_{\mathrm{i}}}{\partial q}$ :

$$
E\left(q, n_{\mathrm{i}}, \frac{\partial n_{\mathrm{i}}}{\partial q}\right)=-\Xi_{0}\left(\frac{e q}{\sqrt{\epsilon_{0} T_{\mathrm{e}} n_{\mathrm{i}}}}\right) \sqrt{\frac{T_{\mathrm{e}} n_{\mathrm{i}}}{\epsilon_{0}}}-\Xi_{1}\left(\frac{e q}{\sqrt{\epsilon_{0} T_{\mathrm{e}} n_{\mathrm{i}}}}\right) \frac{T_{\mathrm{e}}}{e} \frac{\partial n_{\mathrm{i}}}{\partial q}
$$

The uniquely defined functions $\Xi_{0}$ and $\Xi_{1}$ (see fig. 2) are smooth (in fact, analytical) functions of their argument $\xi$ which is the $q$-version of the Debye length-weighted distance from the location to the equivalent electron edge:

$$
\xi=\frac{e q}{\sqrt{\epsilon_{0} T_{\mathrm{e}} n_{\mathrm{i}}}} \equiv \frac{q-0}{\lambda_{\mathrm{D}} n_{\mathrm{i}}} \approx \frac{x-s}{\lambda_{\mathrm{D}}}
$$

Physically, $\Xi_{0}$ and $\Xi_{1}$ act as 'switches' between the depletion and the quasineutral regime. The limit $q \ll 0$ gives $\Xi_{0}=-\xi$ and $\Xi_{1}=0$ and describes the depletion field; the limit $q \gg 0$ yields the ambipolar field with $\Xi_{0}=0$ and $\Xi_{1}=1$. In the thin transition zone $|q| \lesssim \lambda_{\mathrm{D}} n_{\mathrm{i}}$, the AAA is an approximation which is correct up to errors $\sim\left(\lambda_{\mathrm{D}} / l\right)^{2}$. 
Once the AAA is implemented, the rest follows quickly: The ion equation of motion is also transformed into charge coordinates,

$$
-\frac{\Psi_{\mathrm{i}}^{2}}{n_{\mathrm{i}}^{2}} \frac{\partial n_{\mathrm{i}}}{\partial q}=\frac{e}{m_{i}} E+\frac{1}{\lambda} \frac{\Psi_{\mathrm{i}}^{2}}{n_{\mathrm{i}}^{2}} .
$$

Inserting the AAA field expression (7) and defining the functions of $q$ and $n_{\mathrm{i}}$,

$$
\begin{aligned}
L\left(q, n_{\mathrm{i}}\right) & =\frac{T_{\mathrm{e}}}{m_{i}} \Xi_{1}\left(\frac{e q}{\sqrt{\epsilon_{0} T_{\mathrm{e}} n_{\mathrm{i}}}}\right)-\frac{\Psi_{\mathrm{i}}^{2}}{n_{\mathrm{i}}^{2}}, \\
R\left(q, n_{\mathrm{i}}\right) & =\frac{1}{\lambda} \frac{\Psi_{\mathrm{i}}^{2}}{n_{\mathrm{i}}^{2}}-\frac{e}{m_{i}} \Xi_{0}\left(\frac{e q}{\sqrt{\epsilon_{0} T_{\mathrm{e}} n_{\mathrm{i}}}}\right) \sqrt{\frac{T_{\mathrm{e}} n_{\mathrm{i}}}{\epsilon_{0}}},
\end{aligned}
$$

one arrives at the sheath equation, an ordinary differential equation for the ion density $n_{\mathrm{i}}$ as a function of the spatial coordinate $q$.

$$
L\left(q, n_{\mathrm{i}}\right) \frac{\partial n_{\mathrm{i}}}{\partial q}=R\left(q, n_{\mathrm{i}}\right) .
$$

The sheath equation (12) resembles the presheath model (4) but overcomes its deficiency: Its singularity is removable (see the topology in fig. 3): Exactly one physical solution passes through the intersection $\left(q^{*}, n_{\mathrm{i}}^{*}\right)$ of the curves $L\left(q, n_{\mathrm{i}}\right)=0$ and $R\left(q, n_{\mathrm{i}}\right)=0$. Once the ion density $n_{\mathrm{i}}(q)$ is found, the electric field $E(q)$ follows from (7). Also, the transformation back into the $x$-coordinates can be carried out. Then the potential $\Phi(x)$ and the electron density $n_{\mathrm{e}}(x)$ can be calculated. Fig. 4 shows the results for the parameters of the original model. The agreement is excellent, the relative deviation is in the percentage range. (It is a small fraction of $\left(\lambda_{D} / l\right)^{2}$ which is between 0.04 to 0.1 for all solutions.)

The sheath equation (12) achieves what was demanded above: It delivers a simultanous decription of both the ambipolar plasma (for $q \gg q^{*}$ ) and the unipolar sheath (for $q \ll q^{*}$ ), and it was derived without adopting the assumption that the Debye length is small compared to the mean free path. It is interesting to note, however, that the location of the removable singularity which links the branches depends solely on the ratio of those two parameters. For a concise notation, the functional inverse of $\Xi_{0}(\xi) / \Xi_{1}(\xi)$ is defined $\Xi(\cdot)=\left(\Xi_{0} / \Xi_{1}\right)^{-1}(\cdot)$, its functional composition with $\sqrt{\Xi_{1}(\xi)}$ is written $V(\cdot)=\sqrt{\Xi_{1}(\Xi(\cdot))} \equiv \sqrt{\Xi_{1}\left(\left(\Xi_{0} / \Xi_{1}\right)^{-1}(\cdot)\right)}$. Denoting by an asterisk that $\lambda_{\mathrm{D}}^{*}$ and $\lambda^{*}$ are evaluated at the singularity itself, the reduced location $\xi^{*}=e q^{*} / \sqrt{\epsilon_{0} T_{\mathrm{e}} n_{\mathrm{i}}^{*}}$ and the corresponding ion speed $\left|v_{\mathrm{i}}^{*}\right|=\Psi_{\mathrm{i}} / n_{\mathrm{i}}^{*}$ are given as

$$
\begin{aligned}
\xi^{*} & =\Xi\left(\frac{\lambda_{\mathrm{D}}^{*}}{\lambda^{*}}\right), \\
\left|v_{\mathrm{i}}^{*}\right| & =V\left(\frac{\lambda_{\mathrm{D}}^{*}}{\lambda^{*}}\right) \sqrt{\frac{T_{\mathrm{e}}}{m_{\mathrm{i}}}} .
\end{aligned}
$$


The curves are displayed in fig. 5. For small values of $\lambda_{\mathrm{D}}^{*} / \lambda^{*}$, the singularity is located in the quasineutral zone $\left(\xi^{*} \gg 0\right)$ and its speed $\left|v_{\mathrm{i}}^{*}\right|$ is equal to the Bohm speed $v_{\mathrm{B}}=\sqrt{T_{\mathrm{e}} / m_{\mathrm{i}}}$. With growing $\lambda_{\mathrm{D}}^{*} / \lambda^{*}$, the singularity approaches the electron edge $\left(\xi^{*} \approx 0\right)$ and $\left|v_{\mathrm{i}}^{*}\right|$ decreases. In the collisional regime, finally, the singularity is located deep in the depletion zone $\left(\xi^{*} \ll 0\right)$ and $\left|v_{\mathrm{i}}^{*}\right|$ becomes very small. Based on these observations, and on the fact that it directly replaces the essential singularity of the presheath model (4), it is proposed to identify the removable inner singularity $\left(q^{*}, n_{\mathrm{i}}^{*}\right)$ with the sought-after 'collisionally modified Bohm point' and to call its physical characterization - in particular the ion speed condition eq. (14) a 'collisionally modified Bohm criterion'.

Clearly, it is of interest to compare the new criterion with the results obtained by others. Fig. 5 displays the expressions $\left(1+\lambda_{\mathrm{D}} / \lambda\right)^{-1 / 2}\left(\right.$ Godyak [6]), $\left(1+\left(\lambda_{\mathrm{D}} / \lambda\right)^{2 / 3}\right)^{-1 / 2}$ (Valentini [8]), and $\left(1+12.9\left(\lambda_{\mathrm{D}} / \lambda\right)^{0.8}\right)^{-1 / 2}$ (Chen [9]), all proposed as the ratio of a collisionally modified Bohm speed to the standard value. (Formulas converted to the notation of this manuscript.) All curves agree qualitatively with the curve $V\left(\lambda_{\mathrm{D}}^{*} / \lambda^{*}\right)$ presented here; they state that the ion speed at the edge of a collisional sheath lies below the Bohm speed, in a way that solely depends on the ratio of the Debye length $\lambda_{\mathrm{D}}$ to the mean free path $\lambda$. There are, however, considerable quantitative differences. Undoubtedly these differences reflect a certain measure of arbitrariness in the various approaches concerning the 'exact location' of the sheath edge (and possibly also uncertainties concerning the exact definition of $\lambda_{\mathrm{D}}$ and $\lambda$ ).

To what an extent, now, is the sheath criterion proposed in this manuscript not arbitrary, and how does it cope with the cited criticism of Franklin and Riemann? First, the proposed criterion is unambiguous: It relies on the characterization of a well-defined singularity within a well-defined description (10) - (12) of the sheath. Second, the new criterion is consistent: The description (10) - (12) was derived from the standard model (1) - (3) by invoking the advanced algebraic approximation. This in turn rests solely on the assumption $\lambda_{\mathrm{D}} \ll n_{\mathrm{i}} / \frac{\partial n_{\mathrm{i}}}{\partial x}$ which is safely applicable for all ratios $\lambda_{\mathrm{D}} / \lambda$. (Attested by the nearly perfect match of the density curves in figs. 1 and 4.) And third, it is entirely reasonable to call (14) a 'collisionally modified Bohm criterion': As the original Bohm criterion, it marks the transition from the ambipolar diffusion dynamics of the plasma bulk to the unipolar motion in the sheath, and it reduces to its prototype in the collisionless limit. Moreover, the original Bohm point of the presheath equation (4) and the collisionally modified Bohm point of the sheath equation (14) proposed here are in direct mathematical analogy. 
In summary, this manuscript claims that there is a collisionally modified Bohm criterion: Exactly the opposite of what Riemann and Franklin concluded. However, the difference in opinion is only about words, not about physics: Riemann's and Franklin's 'Bohm criterion' and the 'Bohm criterion' of this work rely on different assumptions and are different entities. They are complementary to each other, not contradictory: In particular, Riemann's sheath analysis and Bohm criterion become exact in the limit $\lambda_{\mathrm{D}} / \lambda \rightarrow 0$ but are not applicable for large degrees of collisionality. In contrast, the sheath model of this work relies (as the advanced algebraic approximation itself) on the condition $\lambda_{\mathrm{D}} \ll n_{\mathrm{i}} / \frac{\partial n_{\mathrm{i}}}{\partial x}$, an assumption which is never 'exact' but always 'reasonable', i.e, fulfilled to a satisfactory degree for all ratios $\lambda_{\mathrm{D}} / \lambda$. The same holds for the modified Bohm criterion proposed in this work.

It remains to mention that the approach presented in this manuscript can be extended to RF modulated sheaths. Several investigations which have verified the presented ideas or used them for practical work are already completed [16-24], others - particularly in the field of microplasmas - are being conducted.

Acknowledgment. - The author gratefully acknowledges support by the Deutsche Forschungsgemeinschaft via FOR 1023 and SFB-TR 87.

[1] J. Langmuir, Proc. Natl. Acad. Sci 14, 627 (1928)

[2] L. Tonks and I. Langmuir, Phys. Rev. 34, 876 (1929)

[3] D. Bohm, in The characteristics of electrical discharges in magnetic fields A. Guthrie and R. Wakerling, eds., New York 1949, Chap.3, p.77

[4] K.-B. Persson, Phys. Fluids 5, 1625 (1962)

[5] K.-U. Riemann, J. Phys. D; Appl. Phys. 24, 493 (1991)

[6] V.A. Godyak, Phys. Lett. 89A, 80 (1982)

[7] V.A. Godyak and N. Sternberg, IEEE Trans. Plasma Sci. 18, 159 (1990)

[8] H.-B. Valentini, Phys. Plasmas 3, 1459 (1996)

[9] X.P. Chen, Phys. Plasmas 5, 804 (1997)

[10] V.A. Lisovskiy, 25th IEEE Conf. Plasma Sci. 315 (1998)

[11] K.-U. Riemann, Phys. Plasmas 4, 4158 (1997)

[12] R.N. Franklin, J. Phys. D: Appl. Phys. 36, 2821 (2003) 
[13] R.P. Brinkmann, J. Appl. Phys., 102, 093303 (2007)

[14] R.P. Brinkmann, J. Phys. D: Appl. Phys., 42, 194009 (2009)

[15] V.A. Godyak and Z.K. Ghanna, Sov. J. Plasma Phys. 6, 372 (1979).

[16] M. Kratzer, R.P. Brinkmann, W. Sabisch, and H. Schmidt, J. Appl. Phys. 902169 (2001)

[17] W. Sabisch, M. Kratzer, and R.P. Brinkmann, J. Vac. Sci. Techn. A 21, 1205 (3003)

[18] J. Schulze, B.G. Heil, D. Luggenhölscher, T. Mussenbrock, R.P. Brinkmann, and U. Czarnetzki, J. Phys. D: Appl. Phys. 41, 042003 (2008)

[19] B.G. Heil, J. Schulze, T. Mussenbrock, R.P. Brinkmann, and U. Czarnetzki, IEEE Trans. Plasma Sci. 36, 1404 (2008)

[20] J. Schulze， Z. Donko， B.G. Heil， D. Luggenhölscher， T. Mussenbrock， R.P. Brinkmann， and U. Czarnetzki, J. Phys. D: Appl. Phys. 41, 105214 (2008)

[21] B.G. Heil, U. Czarnetzki, R.P. Brinkmann, T. Mussenbrock, J. Phys. D: Appl. Phys. 41, $165202(2008)$

[22] J. Schulze, B.G. Heil, D. Luggenhölscher, R.P. Brinkmann and U. Czarnetzki J. Phys. D: Appl. Phys. 41, 195212 (2008)

[23] B.G. Heil, R.P. Brinkmann and U. Czarnetzki J. Phys. D: Appl. Phys. 41225208 (2008)

[24] B.G. Heil, R.P. Brinkmann, and U. Czarnetzki J. Phys. D: Appl. Phys. 42, 08520 (2009) 


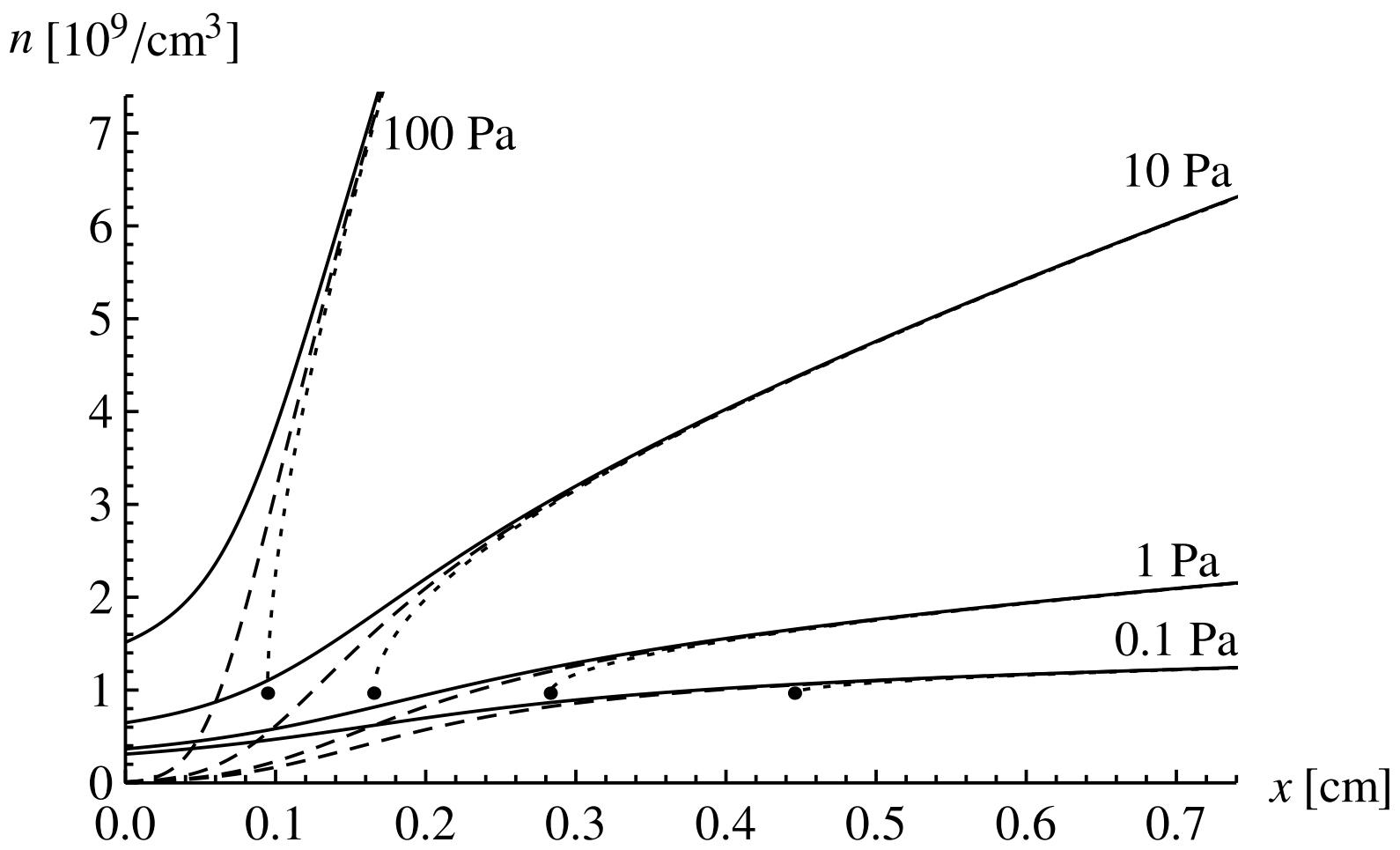

FIG. 1: Densities of the electrons (thin) and ions (thick) in an argon sheath facing a floating wall, for an electron temperature $T_{\mathrm{e}}=3 \mathrm{eV}$ and an ion current $j_{\mathrm{i}}=0.04 \mathrm{~mA} / \mathrm{cm}^{2}$. The neutral gas pressure values are $0.1 \mathrm{~Pa}, 1 \mathrm{~Pa}, 10 \mathrm{~Pa}$, and $100 \mathrm{~Pa}$; corresponding to a nominal scale ratio $\lambda_{D} / \lambda$ of $0.01,0.1,1$, and 10, respectively. Dashed are the plasma solutions which end in a singularity at the conventional Bohm point $v_{\mathrm{B}}=\sqrt{T_{\mathrm{e}} / m_{\mathrm{i}}}=2700 \mathrm{~m} / \mathrm{s}$ and $n_{\mathrm{B}}=9.7 \times 10^{8} \mathrm{~cm}^{-3}$. 


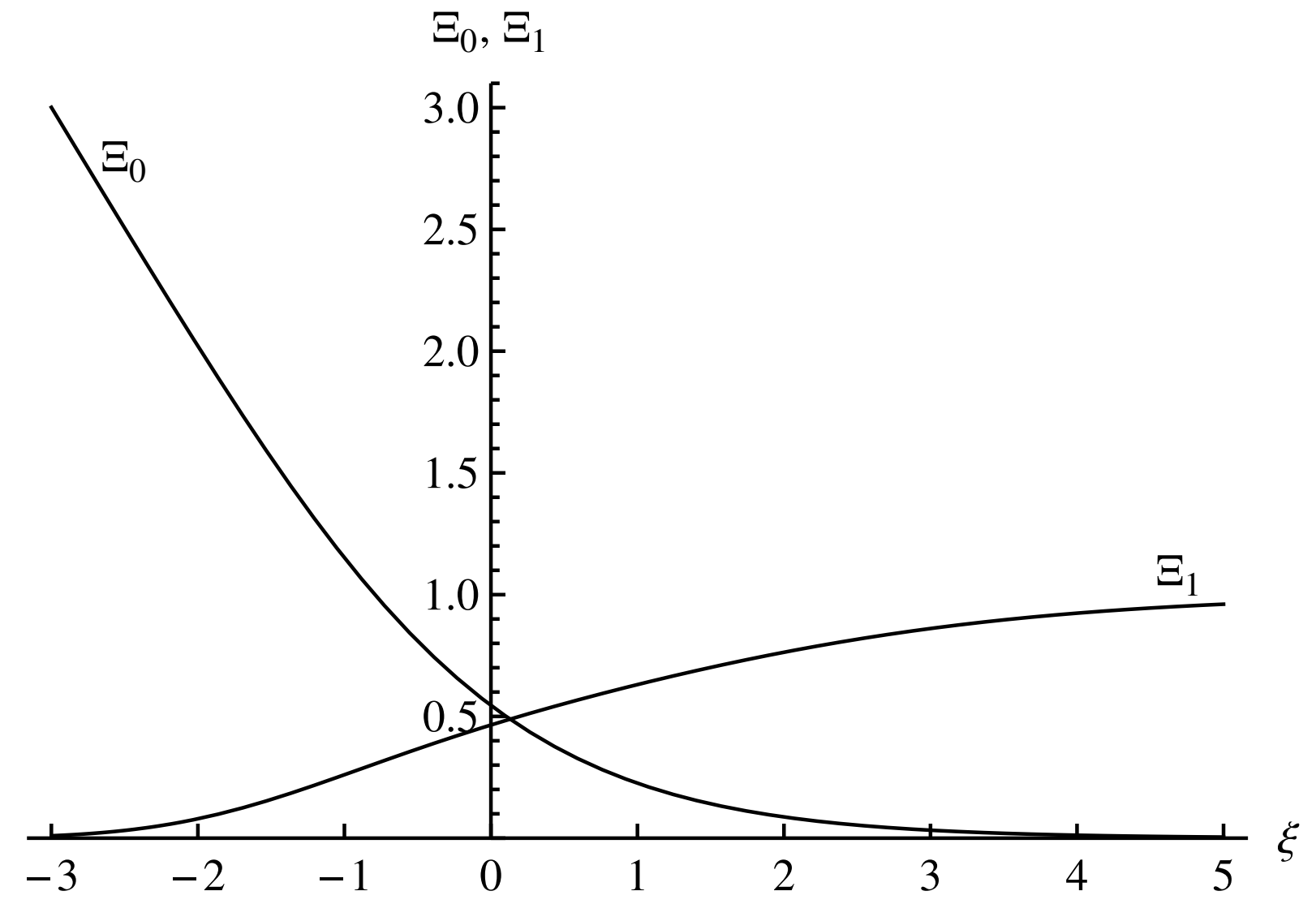

FIG. 2: The analytical switch functions $\Xi_{0}(\xi)$ and $\Xi_{1}(\xi)$ of the advanced algebraic approximation in dependence of their argument $\xi$. 


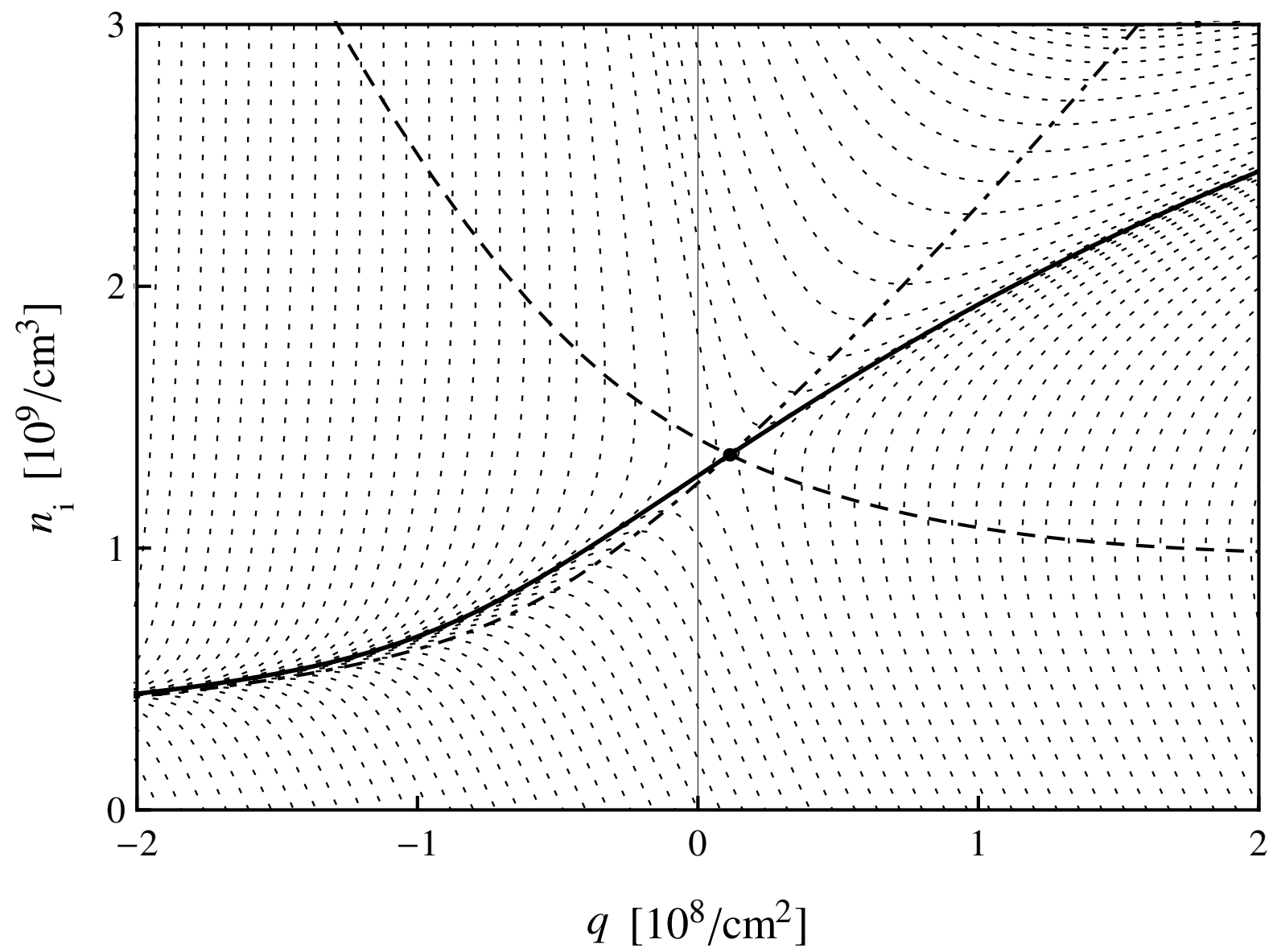

FIG. 3: Phase portrait of the sheath equation at $p=10 \mathrm{~Pa}$. The topology is structured by the dashed curve $L\left(q, n_{\mathrm{i}}\right)=0$ and the dotdashed curve $R\left(q, n_{\mathrm{i}}\right)=0$. Their intersection is a removable inner singularity of saddle point type which is interpreted as the collisionaly modified Bohm point. Of all the possible solutions indicated by the stream, only one is physical, i.e., monotonous and defined for all $q$ (thick curve). 


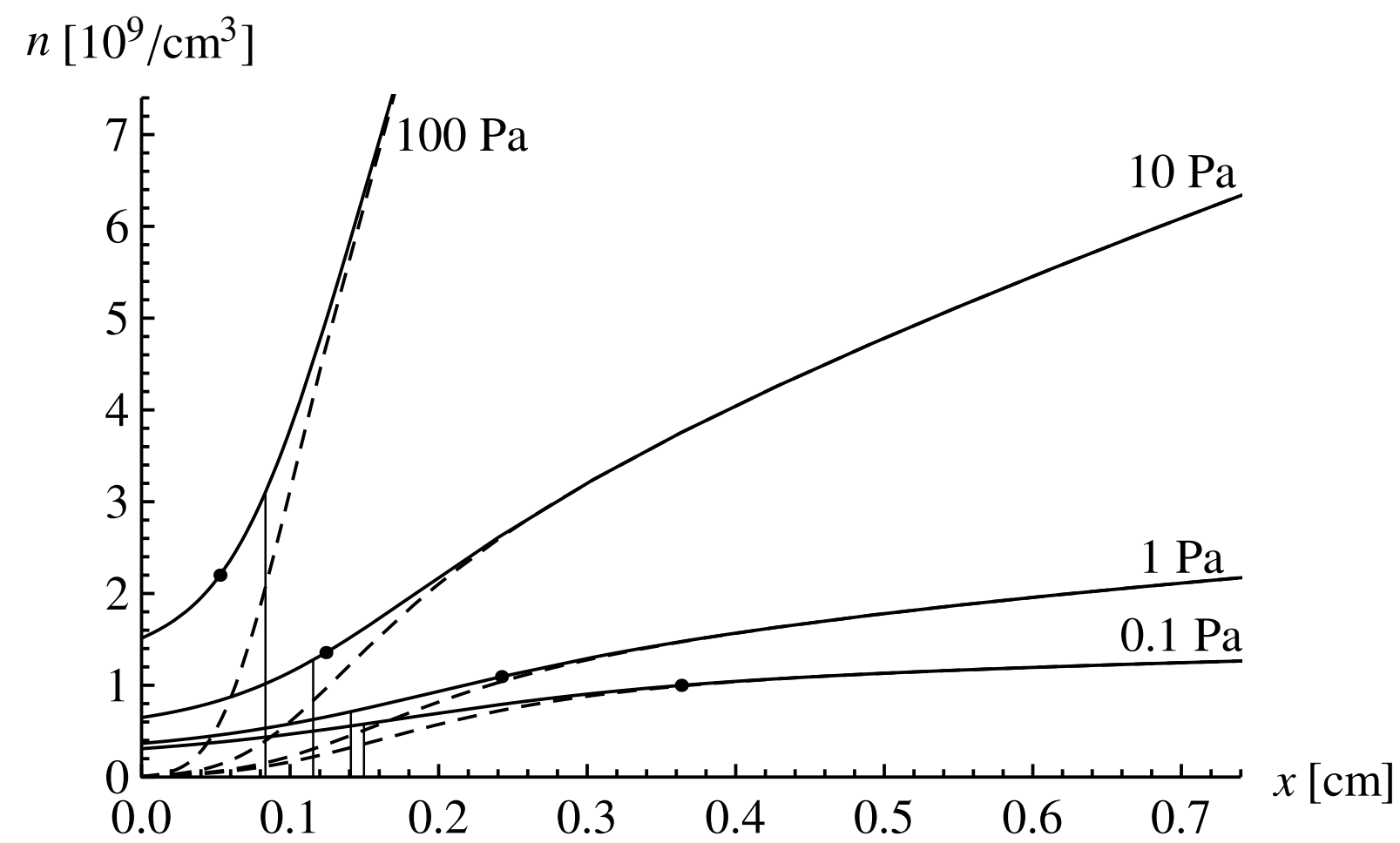

FIG. 4: Density of the electrons (thin) and ions (thick) from the sheath equation, for the same parameters as the original sheath model (fig. 1). The deviation is less than the width of the lines. The vertical lines indicate the electron edge $s$. The solid points denote the generalized Bohm point defined in this manuscript, i.e., the location of inner singularity of the sheath equation. 


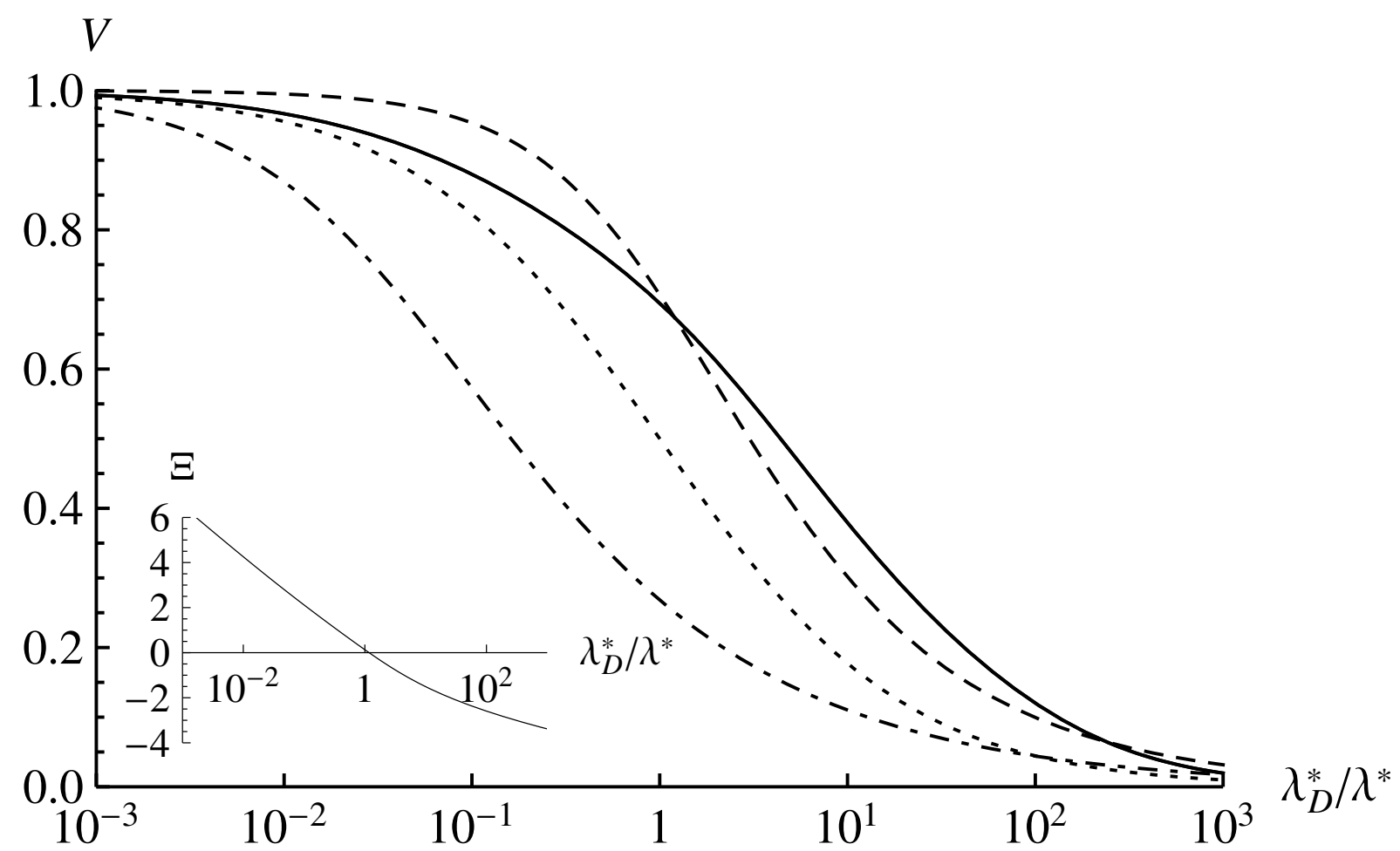

FIG. 5: The ratio $V$ of the collisionally modified Bohm speed to the standard Bohm speed $v_{\mathrm{B}}$ as a function of $\lambda_{\mathrm{D}}^{*} / \lambda^{*}$ (solid), in comparison with the formulas presented by Godyak [6] (dashed), Valentini [8] (dotted), and Chen [9] (dotdashed). The inset shows the location $\Xi$ of the collisionally modified Bohm point as a function of $\lambda_{\mathrm{D}}^{*} / \lambda^{*}$. 